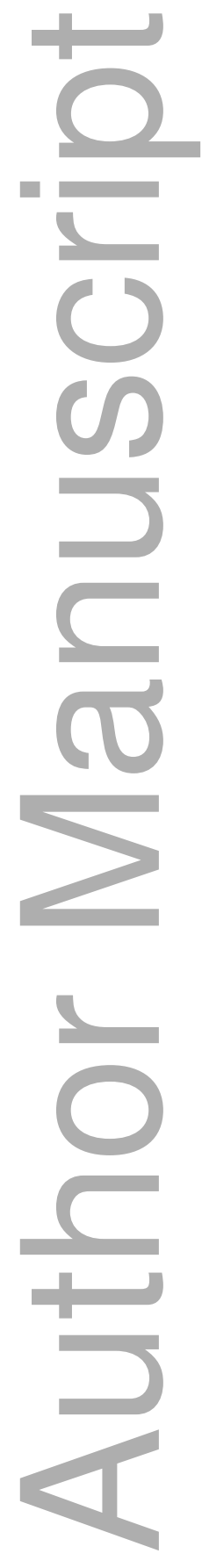

This is the author manuscript accepted for publication and has undergone full peer review but has not been through the copyediting, typesetting, pagination and proofreading process, which may lead to differences between this version and the Version of Record. Please cite this article as doi: $\underline{10.1002 / M R M .27993}$

This article is protected by copyright. All rights reserved 


\title{
Compressed Sensing effects on quantitative analysis of undersampled human brain sodium MRI
}

\author{
Yasmin Blunck ${ }^{1,2 *}$ | Scott C. Kolbe ${ }^{2}$ | Bradford A. \\ Moffat $^{2}$ | Roger J. Ordidge ${ }^{2}$ | Jon O. Cleary ${ }^{3 \dagger}$ | \\ Leigh A. Johnston ${ }^{1,2 \dagger}$
}

${ }^{1}$ Department of Biomedical Engineering, The University of Melbourne, Parkville,

Australia

2 Melbourne Brain Centre Imaging Unit, Department of Medicine and Radiology, The University of Melbourne, Parkville, Australia

${ }^{3}$ Department of Radiology, Guy's and St. Thomas' NHS Foundation Trust, London, U.K.

\section{Correspondence}

Yasmin Blunck, Melbourne Brain Centre Imaging Unit, Kenneth Myer Building, University of Melbourne, 30 Royal Parade, Parkville VIC 3052, Australia

Email: bluncky@unimelb.edu.au

\section{Funding information}

Bradford Moffat is supported by the Australian National Imaging Facility (NIF).

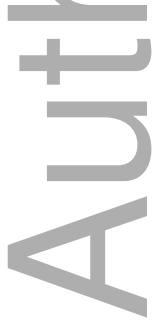

Purpose The clinical application of sodium MRI is hampered due to relatively low image quality and associated long acquisition times. Compressed Sensing (CS) aims at a reduction of measurement time, but has been found to encompass quantitative estimation bias when used in low SNR $x$-Nuclei imaging. This work analyses CS in quantitative human brain sodium MRI from undersampled acquisitions and provides recommendations for tissue sodium concentration (TSC) estimation.

Methods CS reconstructions from 3D radial acquisitions of five healthy volunteers were investigated over varying undersampling factors (USFs) and CS penalty weights on different sparsity domains, Wavelet, Discrete Cosine Transform (DCT) and Identity. Resulting images were compared with highly-sampled and undersampled NUFFT-based images and evaluated for image quality (i.e. structural similarity), image intensity bias and its effect on TSC estimates in gray and white matter.

Results Wavelet-based CS reconstructions show highest image quality with stable TSC estimates for most USFs. Up to an USF of 4, images showed good structural detail. DCT and Identity-based CS enable good image quality, however show a bias in TSC with a reduction in estimates across USFs.

Conclusion The image intensity bias is lowest in Wavelet-based reconstructions and enables an up to four-fold acquisition speed up while maintaining good structural detail. The associated acquisition time reduction can facilitate a translation of sodium MRI into clinical routine.

KEYWORDS

sodium $\left({ }^{23} \mathrm{Na}\right) \mathrm{MRI}, \mathrm{x}$-Nuclei, Compressed Sensing, Iterative Reconstruction, quantitative imaging

\footnotetext{
$\dagger$ Joint senior authors.
} 


\section{1 | INTRODUCTION}

The investigation of MR-observable nuclei in vivo provides powerful means for the diagnosis and study of pathologies. Sodium $\left({ }^{23} \mathrm{Na}\right)$, the nucleus studied in this work, provides the second strongest MR signal in biological tissue and is of great significance in physiology. Healthy cells maintain a well-balanced ion concentration gradient between intra- and extra-cellular space. Pathologies like ischemia, cancer or other tissue damage disrupt this ion-homeostasis leading to raised tissue sodium [1]. Sodium MRI promises insight into these pathological processes and offers potential as a quantitative clinical biomarker with tissue sodium concentration (TSC) being the most frequently studied parameter. Its potential for clinical applications ranges from diagnostic capabilities, extending 'standard' MRI to the detection of pathologies without apparent structural abnormalities, to therapy, including treatment monitoring and drug development [2].

Sodium MRI is primarily limited by two factors: 1) low NMR sensitivity and in vivo concentration and 2) fast biexponential transverse signal decay. The former leads to poor SNR in sodium images, the latter requires dedicated, usually non-Cartesian acquisition schemes. Consequently, sodium MRI suffers from long acquisition times and relatively poor image SNR and resolution. So far these challenges have been primarily addressed through acquisition strategy improvements with regards to sampling efficiency and homogeneity \begin{tabular}{l|l|l|l|l|l|}
3 & 5 & 6 & 7 & as well as sequence timing
\end{tabular} optimization [8]. This work investigates acquisition efficiency improvement by exploiting sparse sampling reconstruction principles.

Compressed Sensing (CS) is a technique for iterative reconstruction from highly undersampled data based on sparsity and incoherence, i.e. noise-like, undersampling artifacts [9 10 11, 12]. The nonlinear nature of CS reconstructions has been shown to achieve high image quality with significant noise suppression [11 13]. This denoising property together with the data acquisition reduction from undersampling make CS an enticing method for sodium MRI.

The potential of CS has been widely studied in ${ }^{1} \mathrm{H}$-based MRI, but so far only a few studies explored its applicability in x-Nuclei imaging, e.g. helium-3 [14, fluorine-19 [15 16], carbon-13 [17] and sodium-23 [18 19 [20| 21 [2]. Previous studies of iterative reconstruction in sodium MRI investigated human knee [18], breast [22] and brain imaging [19 20, 21] with a focus on image quality and structural detail. The utilized CS reconstruction focused on image sparsity [18, ${ }^{1} \mathrm{H}$-based anatomical constraints $\left.19,20,22\right]$ and sparsity in learned dictionaries [21]. While promising potential for SNR improvements [19 20,21 and acquisition time reduction [18] were shown, inadequate regularization constraints and high undersampling (USF $\geq 3$ ) were found to introduce an image intensity error and biased TSC estimation, respectively 18,20 .

With an emphasis on image intensity reliability, a fundamental requirement for bias-free estimation of quantitative parameters in sodium MRI, this work focuses on TV-regularized sparsity CS methods from 3D radial acquisitions in the human brain. Utilizing all common sparsity domains (image domain, discrete cosine and Wavelet transform) over a wide range of undersampling factors we investigate the achievable image quality and perform a correlation analysis of CS reconstructions with highly-sampled acquisitions to evaluate its effect on quantitative parameter estimation.

\section{2 | METHODS}

\section{1 | MR measurements}

Brain imaging of five healthy volunteers (three female, two male, mean age 33.2yrs, range 27-41) was performed on a research 7T MRI scanner (Siemens Healthcare, Erlangen, Germany) with a transmit/receive dual-tuned ${ }^{1} \mathrm{H}-{ }^{23} \mathrm{Na}$ head 
coil (QED, Mayfield Village, Ohio, USA). The human imaging was conducted with the approval of the University of Melbourne Human Research Ethics Committee and volunteers gave informed consent prior to the experiment.

Sodium images were acquired with a custom-built 3D radial sequence based on Saff and Kuijlaars' method [23] for a homogeneous projection placement. Sodium-density weighted images were acquired with the following parameters: $90^{\circ}$ flip angle, repetition time (TR) of $160 \mathrm{~ms}$ and echo time (TE), defined as the time interval between the center of the pulse and sampling onset, was set to $0.4 \mathrm{~ms}$. Measurements were taken with $\mathrm{N}=10,000$ projections and a readout duration ( $T_{R O}$ ) of $2 \mathrm{~ms}$ per projection with a nominal isotropic resolution of $3.1 \mathrm{~mm}$ over a field of view (FOV) of $20 \mathrm{~cm}$. The total acquisition time (TA) was $26 \mathrm{~min} 20 \mathrm{sec}$. For brain region investigations, an additional ${ }^{1} \mathrm{H}$-based FLASH image was acquired with $\mathrm{TR}=11 \mathrm{~ms}, \mathrm{TE}=3.06 \mathrm{~ms}, 14^{\circ}$ flip angle, $1 \mathrm{~mm}$ isotropic resolution and a total acquisition time of $6 \mathrm{~min} 12 \mathrm{sec}$. Brain tissue segmentation was based on a ${ }^{1} \mathrm{H}-\mathrm{MP} 2 \mathrm{RAGE}$ T1-weighted image acquired at inversion times (TI) 700 and 2,700 ms; with $5^{\circ}$ and $6^{\circ}$ flip angle; TR $=4,900 \mathrm{~ms}$; TE $=2.94 \mathrm{~ms}$; GRAPPA factor 4; $0.9 \mathrm{~mm}$ isotropic resolution; and acquisition time $6 \mathrm{~min} .{ }^{1} \mathrm{H}-\mathrm{MP} 2 \mathrm{RAGE}$ were imaged using a 32-channel head coil (Nova Medical Inc., Wilmington MA, USA) in a separate imaging session.

$\mathrm{B}_{0}$-field shimming was performed with a ${ }^{1} \mathrm{H}$-based vendor-provided 3D shimming routine. Shim optimization was repeated 10 times prior to image acquisition.

\section{2 | Image Reconstruction}

CS-based reconstructions generally consist of two components: data consistency (1) in Eq 1 and additional regularization terms (2) in Eq 1, which commonly exploit transform sparsity or other a priori information [19 20 22]. The applied optimization problem underlying this work focuses on the conventional CS elements [11] and can be formulated as:

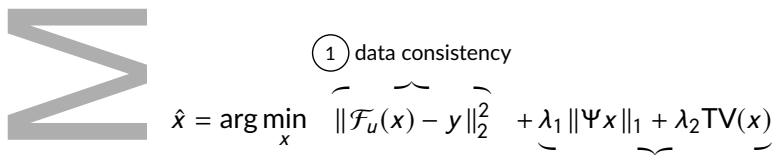

(2) regularization

where $\|\cdot\|_{2}$ and $\|\cdot\|_{1}$ denote the $I_{1}$ and $I_{2}$-norm, respectively, $\mathcal{F}_{u}$ is the undersampled NUFFT-operator, $x$ is the reconstructed image, $y$ the undersampled raw data, $\psi$ the applied sparsity transform and $\lambda_{1}$ and $\lambda_{2}$ the weights on the transform sparsity and total variation (TV), respectively.For an analysis of regularization terms on overall reconstruction results, $\lambda_{1}$ and $\lambda_{2}$ have been tested over a range of weightings: $\lambda_{1}$ (sparsity domain) $=[0,1.0]$ step size $0.1 ; \lambda_{2}(\mathrm{TV}$ ) $=[0,0.0001,0.0005,0.001,0.005,0.01,0.05,0.1,0.5]$.

Image reconstruction was performed offline in MATLAB (Mathworks, Natick, MA, USA) with a Nonuniform Fast Fourier Transform (NUFFT) 24] provided in the Michigan Image Reconstruction Toolbox (MIRT). Images were reconstructed onto an isotropic resolution of $3.1 \mathrm{~mm}$. Prior to reconstruction, projection raw data was compensated for density inhomogeneities as described by Nielles-Vallespin et al. [25]. Furthermore, a Hann filter was applied to reduce Gibbs' ringing artifacts. To compensate the lower number of projections in undersampled reconstructions, $N_{u}$, an additional weighting factor $\left(N / N_{u}\right)$ was applied to the raw data to account for the degree of undersampling. It should be noted that the data consistency term in Equation 1 encompasses all raw data weighting. The described optimization problem was solved with a MATLAB-based nonlinear conjugate gradient method [11] over 50 iterations.

For the investigation and evaluation of undersampling effects on image quality and estimation random projections were omitted from the acquired raw data and the undersampled raw data was reconstructed with 1) standard NUFFT 
and 2) iterative CS reconstruction with all possible combinations of described regularization weights and sparsity domains. Undersampling factors (USFs) ranged from 2 ( $50 \%$ original data) to 10 (10\% of original data) with increments of 1 .

\section{3 | Image Analysis}

Brain regions were extracted from ${ }^{1} \mathrm{H}$-based acquisitions and linearly co-registered to the sodium images. Specifically, the FLASH image was used for a brain mask extraction and MP2RAGE served for the segmentation of specific brain compartments, namely cerebrospinal fluid (CSF), gray matter (GM) and white matter (WM). All described image processing steps were performed in FSL using FLIRT, BET and FAST, respectively 26. The downsampling of the high resolution tissue masks to the lower sodium image resolution was performed with a tri-linear interpolation. The resulting partial volume maps were thresholded at 0.8 to reduce partial volume contamination in the tissue sodium concentration (TSC) estimation.

Reconstruction performance for all undersampled sodium reconstructions was assessed using structural similarity (sSim) scores with $\mathrm{I}_{\text {ref }}$. SSim measures were only calculated over the brain region to avoid the influence of background noise. Best performing (i.e. highest sSim score) CS-based and standard NUFFT-based image reconstructions of undersampled data $\left(\mathrm{I}_{\mathrm{u}}\right)$ were compared to the original, highly sampled $\left(10,000\right.$ projections) reference image $\left(\mathrm{I}_{\text {ref }}\right)$ for an evaluation of image intensity accuracy via a pixel-wise correlation. The correlation analysis was performed in MATLAB on normalized $I_{\text {ref }}$ and $I_{u}$, i.e. each image was individually scaled by its maximum intensity to ensure a comparable dynamic range up to 1 .

Mean TSC values were calculated for GM and WM in all undersampled reconstructions, i.e. NUFFT and CS-based reconstructions with highest $\mathrm{SS}$ Sim score and compared with TSC estimates from Iref. TSC was calculated as a linear calibration between the mean background signal $(0 \mathrm{mmol} / \mathrm{l})$ and the mean CSF signal ( $145 \mathrm{mmol} / \mathrm{I}$ [27]) in one subject where the CSF ROI could be placed such that partial volume contamination was avoided. The CSF ROI spanned $2 \times 2$ voxels in the transverse plane and was manually positioned in the right lateral ventricle. The mean CSF signal was scaled by a factor of $1 /[1-\exp (-\mathrm{TR} / \mathrm{T} 1)]$ with $\mathrm{T} 1=50 \mathrm{~ms}$ to compensate T1-weighting [28]. As outlined above, quantification of TSC was performed on GM and WM masks with a minimal partial volume contribution of 0.8 as obtained from the linear co-registration of MP2RAGE tissue segmentation masks.

\section{3 | RESULTS}

Wavelet-based CS reconstructions achieve high sSim scores over a range of investigated regularization weights (Figure 1). The sSim analysis suggests that the optimal regularization weights are independent of the USF for all investigated sparsifying transforms. It is to be expected that iterative CS reconstructions improve image quality compared to standard NUFFT reconstructions, however, an appropriate weighting of regularization weights is essential, particularly for identity-based CS reconstructions (c.f. red surrounded area in Figure 1). Achievable image quality, i.e. sSim, decreases with higher USF. However, from visual inspection structural features were found to be well preserved up to an USF of 4 (Supporting Figure S.1). Henceforth, any $I_{u}$ describes the reconstruction result that achieved the highest sSim score for a respective sparsity domain and USF.

The intensity correlation analysis between $I_{r e f}$ and $I_{u}$ reveals an increasing deviation, i.e. wider point cloud spread, with higher USF (Figure 2; Supporting Figure S.2 provides a detailed overview in a single subject). Linear model fits produce slopes that decrease with increasing USF, particularly for NUFFT and Identity-based CS reconstructions, 
suggestive of a reduction in the overall dynamic range, that is an increase in mean noise and decrease in high image intensities. Wavelet-based reconstructions produce linear fits, that are overall closest to the ideal $I_{\text {ref }}=I_{u}$ line (black dashed line in Figure 2) and show residuals randomly distributed around zero. Particularly, DCT and Identity-based CS reconstructions show a slight bias in their residuals suggesting a non-linear point cloud distribution. Furthermore, the linear fits for Wavelet-based reconstructions achieve highest $r^{2}$ values for all USF Table 1 .

Observing the mean signal within image intensity intervals further highlights the nonlinear bias in intensity reconstructions (Figure 3). Here the normalized $I_{\text {ref }}$ was divided into 200 voxel groups that were categorized according to their image intensity, each voxel group spanning an intensity region of the same width (i.e. $1 / 200=0.005$ ). A correlation of the mean signal in $\mathrm{I}_{\text {ref }}$ and $\mathrm{I}_{\mathrm{u}}$ in each voxel group shows that the mean noise increase and high signal suppression builds up with higher USF. A focus on the lower intensity region (red solid box in Figure 3) provides evidence of the better noise suppression in iterative CS reconstructions. Wavelet-based reconstructions lead to the smallest drop in high signal intensities (blue dashed box in Figure 4).

The effects on the intensity range yield consequences for TSC estimates in gray and white matter (Figure 4). For DCT and Identity-based CS reconstructions TSC estimates generally fall below TSC estimates from Iref. A decrease in TSC estimates is also observed in undersampled NUFFT reconstructions. For moderate undersampling, i.e. USF < 4, the TSC underestimation is smaller than in DCT and Identity-based CS results. Wavelet-based CS reconstructions achieve TSC estimates that are comparable with $\mathrm{I}_{\text {ref }}$ TSC estimates for most USFs.

\section{4 | DISCUSSION}

The quantitative capability of sodium MRI is one of its strengths. Clinical studies of sodium MRI commonly focus on the evaluation of tissue sodium concentration (TSC), a marker well known to be linked to tissue viability [1] and with promising scan-rescan robustness 29 . The quantification of sodium content is based on signal intensities from known sodium concentrations in sodium-density weighted acquisitions. Hence, for a reliable estimation of TSC, accurate signal intensities are essential. The question of image intensity reconstruction performance in sodium MRI CS has not previously been studied in detail. A reduction in TSC estimates from undersampled CS reconstructions have, however, been reported [18. Underestimation effects were also described in quantitative assessment of CS reconstructions in ${ }^{19} \mathrm{~F}$ MRI [15]. Since the mean background noise signal is commonly used as a $0 \mathrm{mmol} / \mathrm{l}$ marker, the denoising effect of TV-regularized CS can lead to a problematic bias for quantitative assessments. The $I_{1}$-norm for sparsity terms may have signal magnitude shrinking effects 11 . Correspondingly, a decrease of high image intensities has been observed in this study. Together these effects have been shown to lead to a potential underestimation of TSC values, particularly for suboptimal sparsity transforms (DCT and Identity).

Noise plays a significant role in the robustness of TSC estimation. The reduction in SNR from undersampling also affects estimation results in standard NUFFT reconstructions with stronger underestimation at higher USFs. Noise in the image domain can be successfully mitigated through CS reconstruction. For higher USFs, the bias in TSC estimation is therefore smaller in CS reconstructions compared with NUFFT images. For lower USFs (USF $\leq 3$ ), however, NUFFT show a slightly lower bias than DCT and Identity-based CS reconstructions. The denoising effect is a well known benefit of CS in X-Nuclei MRI [18 $15,19,20,21,16$, 16 and originates from the nature of TV-regularized nonlinear reconstruction. Unlike standard NUFFT-based magnitude noise profiles which follow a well-known Rayleigh distribution, the statistical characteristics of noise from iterative reconstruction are still not fully understood. The effects on statistical and spatial noise distribution may however bias the estimation of other parameters such as $T_{2}$ 30 31. 32. 
The bias in intensity reconstruction is predominantly found at low and high intensities (Figure 3). This suggests that the use of a range of sodium concentration phantoms across the whole spectrum of brain sodium concentrations (20 - $150 \mathrm{mmol} / \mathrm{l}$ 27]) can mitigate the bias in quantitative estimates from CS reconstructed images; the use of background signal as a $0 \mathrm{mmol} / \mathrm{l}$ reference should be avoided for an unbiased estimation. The aspect of feature size in signal intensity bias of CS reconstructions has not been studied in this work, however, an increased bias for small features has been reported for ${ }^{19} \mathrm{~F}$ MRI 15 .

The Wavelet transform is well known to be a robust method for the compression of natural images [33] and, hence, not surprisingly showed the best performance in this study. The optimal reconstruction penalties and respective weightings depend on the SNR of the acquired raw data and the complexity of the imaged object [13]. Recent work on the application of standard CS in sodium MRI of the human knee showed sufficient sparsity in the image domain 18. For sodium MRI of the human brain, as studied in this work, the identity transform was found to be an insufficient sparsity domain. This finding highlights the need for dedicated studies of CS in specific MRI applications as CS reconstruction is strongly problem-dependent and not necessarily generalizable. It should be noted that the investigated quality parameter in this study (sSim) assumes the reference image to be the perfect ground truth. For in vivo studies such information is not readily available and, hence, a highly sampled standard NUFFT reconstruction was used instead. Reconstruction quality parameters were only evaluated within the individual brain mask to mitigate the influence of noise in reconstruction quality assessment.

This work has addressed the foundational components of CS algorithms, transform sparsity and TV regularization. It should be noted that there exists a variety of CS methods such as low-rank reconstructions 34 and dictionary learning approaches [35. These CS methods achieve image enhancement characterized by a suppression of noise while also maintaining structural details. Although these modern CS algorithms achieve reconstruction results that are visually indifferentiable from Nyquist sampled acquisitions, highest reconstruction errors are commonly found around high image intensities 34 35. These characteristics suggest that the bias in intensity extremes from iterative reconstruction of undersampled data, as analysed in the current work, persists regardless of the CS algorithm employed.

Previous studies on iterative reconstructions in sodium MRI of the human brain investigated the incorporation of ${ }^{1} \mathrm{H}$-based anatomical information 19,20 and dictionary-based approaches [21]. These studies demonstrated that the inclusion of sparsity in learned dictionaries or anatomical constraints improve SNR and maintain good structural detail. Iterative reconstruction is not limited in the number of penalty terms, and anatomical information as well as dictionaries could easily be added to the CS cost function that was investigated in this work.

The number of radial projections is a strongly varying parameter across studies. Since radial raw data can be reconstructed onto any desired FOV, the number of projections is often chosen as a trade-off between total acquisition time and image quality. The results from this study should therefore rather be interpreted as a total number of spokes (USF $4=2,500$ spokes) and support the conclusion that CS reconstructions with optimized parameters can resemble images with 4-times more densely sampled raw data.

\section{5 | CONCLUSION}

Driven by an interest in acquisition time reduction, this work investigated the effect of TV-regularized sparsity CS reconstructions on image intensities in sodium MRI of the human brain. The image intensity bias from undersampling was best controlled in Wavelet-based CS, resulting in stable TSC estimates. This well-known sparsity transform was shown to enable undersampling of up to a factor of 4 with high image quality and structural detail while maintaining good signal intensity reconstruction and estimation performance. 


\section{acknowledgements}

We acknowledge the facilities, and the scientific and technical assistance of the Australian National Imaging Facility at the Melbourne Brain Centre Imaging Unit. We appreciate the high performance computing facility for imaging and visualization provided by the Multi-modal Australian ScienceS Imaging and Visualisation Environment (MASSIVE). The work was also supported by a research collaboration agreement with Siemens Healthcare. The MP2RAGE data was acquired with a prototype sequence provided by Siemens Healthcare. Bradford Moffat is supported by the Australian National Imaging Facility (NIF).

\section{references}

[1] Ouwerkerk R. Sodium MRI. In: Magnetic Resonance Neuroimaging Springer; 2011.p. 175-201.

[2] Thulborn KR. Quantitative sodium MR imaging: A review of its evolving role in medicine. Neuroimage 2016;168:250268.

[3] Boada FE, Gillen JS, Shen GX, Chang SY, Thulborn KR. Fast three dimensional sodium imaging. Magnetic Resonance in Medicine 1997;37(5):706-15.

[4] Nagel AM, Laun FB, Weber MA, Matthies C, Semmler W, Schad LR. Sodium MRI using a density-adapted 3D radial acquisition technique. Magnetic Resonance in Medicine 2009;62(6):1565-73.

[5] Lu A, Atkinson IC, Claiborne TC, Damen FC, Thulborn KR. Quantitative sodium imaging with a flexible twisted projection pulse sequence. Magnetic Resonance in Medicine 2010;63(6):1583-93.

[6] Riemer F, Solanky BS, Stehning C, Clemence M, Wheeler-Kingshott CA, Golay X. Sodium 23Na ultra-short echo time imaging in the human brain using a 3D-Cones trajectory. MAGMA 2014;27(1):35-46.

[7] Stobbe R, Beaulieu C. Advantage of sampling density weighted apodization over postacquisition filtering apodization for sodium MRI of the human brain. Magnetic Resonance in Medicine 2008;60(4):981-6.

[8] Blunck Y, Moffat BA, Kolbe SC, Ordidge RJ, Cleary JO, Johnston LA. Zero-gradient-excitation ramped hybrid encoding (zGRF-RHE) sodium MRI. Magnetic Resonance in Medicine 2019;81(2):1172-1180.

[9] Donoho DL. Compressed Sensing. IEEE Transactions on Information Theory 2006;52(4):1289-1306.

[10] Candès EJ, Romberg J, Tao T. Robust uncertainty principles: Exact signal reconstruction from highly incomplete frequency information. IEEE Transactions on Information Theory 2006;52(2):489-509.

[11] Lustig M, Donoho D, Pauly JM. Sparse MRI: The application of compressed sensing for rapid MR imaging. Magnetic Resonance in Medicine 2007;58(6):1182-95.

[12] Candès EJ, Wakin MB. An Introduction to Compressive Sampling. IEEE Signal Processing Magazine 2008;25(2):21-30.

[13] Block KT, Uecker M, Frahm J. Undersampled radial MRI with multiple coils. Iterative image reconstruction using a total variation constraint. Magnetic Resonance in Medicine 2007;57(6):1086-98.

[14] Ajraoui S, Lee KJ, Deppe MH, Parnell SR, Parra-Robles J, Wild JM. Compressed Sensing in hyperpolarized 3 He lung MRI. Magnetic Resonance in Medicine 2010;63(4):1059-69.

[15] Zhong J, Mills PH, Hitchens TK, Ahrens ET. Accelerated fluorine-19 MRI cell tracking using compressed sensing. Magnetic Resonance in Medicine 2013;69(6):1683-90.

\section{This article is protected by copyright. All rights reserved}


[16] Liang S, Dresselaers T, Louchami K, Zhu C, Liu Y, Himmelreich U. Comparison of different compressed sensing algorithms for low SNR 19F MRI applications-Imaging of transplanted pancreatic islets and cells labeled with perfluorocarbons. NMR in Biomedicine 2017;30(11):e3776.

[17] Hu S, Lustig M, Chen AP, Crane J, Kerr A, Kelley DA, et al. Compressed Sensing for resolution enhancement of hyperpolarized 13C flyback 3D-MRSI. Journal of Magnetic Resonance 2008;192(2):258-64.

[18] Madelin G, Chang G, Otazo R, Jerschow A, Regatte RR. Compressed sensing sodium MRI of cartilage at 7T: preliminary study. Journal of Magnetic Resonance 2012;214(1):360-5.

[19] Gnahm C, Bock M, Bachert P, Semmler W, Behl NG, Nagel AM. Iterative 3D projection reconstruction of 23Na data with an 1H MRI constraint. Magnetic Resonance in Medicine 2014;71(5):1720-32.

[20] Gnahm C, Nagel AM. Anatomically weighted second-order total variation reconstruction of 23Na MRI using prior information from 1H MRI. Neuroimage 2015;105:452-61.

[21] BehI NG, Gnahm C, Bachert P, Ladd ME, Nagel AM. Three-dimensional dictionary-learning reconstruction of 23Na MRI data. Magnetic Resonance in Medicine 2016;75(4):1605-16.

[22] Lachner S, Zaric O, Utzschneider M, Minarikova L, Zbỳň Š, Hensel B, et al. Compressed sensing reconstruction of 7 Tesla 23Na multi-channel breast data using 1 H MRI constraint. Magnetic resonance imaging 2019;60:145-156.

[23] Saff EB, Kuijlaars AB. Distributing many points on a sphere. The Mathematical Intelligencer 1997;19(1):7.

[24] Fessler JA, Sutton BP. Nonuniform fast Fourier transforms using min-max interpolation. IEEE Transactions on Signal Processing 2003;51(2):560-574.

[25] Nielles-Vallespin S, Weber MA, Bock M, Bongers A, Speier P, Combs SE, et al. 3D radial projection technique with ultrashort echo times for sodium MRI: clinical applications in human brain and skeletal muscle. Magnetic Resonance in Medicine 2007;57(1):74-81.

[26] Smith SM, Jenkinson M, Woolrich MW, Beckmann CF, Behrens TE, Johansen-Berg H, et al. Advances in functional and structural MR image analysis and implementation as FSL. Neuroimage 2004;23 Suppl 1:S208-19.

[27] Madelin G, Lee JS, Regatte RR, Jerschow A. Sodium MRI: methods and applications. Progress in Nuclear Magnetic Resonance Spectroscopy 2014;79:14-47.

[28] Qian Y, Zhao T, Zheng H, Weimer J, Boada FE. High-resolution sodium imaging of human brain at 7 T. Magnetic resonance in medicine 2012;68(1):227-233.

[29] Riemer F, McHugh D, Zaccagna F, Lewis D, McLean MA, Graves MJ, et al. Measuring Tissue Sodium Concentration: Cross-Vendor Repeatability and Reproducibility of 23Na-MRI Across Two Sites. Journal of Magnetic Resonance Imaging 2019 https://doi.org/10.1002/jmri.26705

[30] Blunck Y, Josan S, Taqdees SW, Moffat BA, Ordidge RJ, Cleary JO, et al. 3D-Multi-Echo Radial Imaging of $23 \mathrm{Na}$ (3D-MERINA) for time-efficient multi-parameter tissue compartment mapping. Magnetic Resonance in Medicine 2017;79(4):1950-1961.

[31] Zibetti MVW, Baboli R, Chang G, Otazo R, Regatte RR. Rapid compositional mapping of knee cartilage with compressed sensing MRI. J Magn Reson Imaging;48(5):1185-1198.

[32] Syeda W, Blunck Y, Kolbe S, Cleary JO, Johnston LA. A continuum of $T^{*} 2$ components: Flexible fast fraction mapping in sodium MRI. Magnetic Resonance in Medicine 2019;81(6):3854-3864.

[33] Taubman D, Marcellin M. JPEG2000 image compression fundamentals, standards and practice: image compression fundamentals, standards and practice, vol. 642. Springer Science \& Business Media; 2012.

\section{This article is protected by copyright. All rights reserved}


[34] Jin $\mathrm{KH}$, Lee D, Ye JC. A general framework for compressed sensing and parallel MRI using annihilating filter based low-rank Hankel matrix. IEEE Transactions on Computational Imaging 2016;2(4):480-495.

[35] Ravishankar S, Bresler Y. MR image reconstruction from highly undersampled k-space data by dictionary learning. IEEE Transactions on Medical Imaging 2010;30(5):1028-1041.

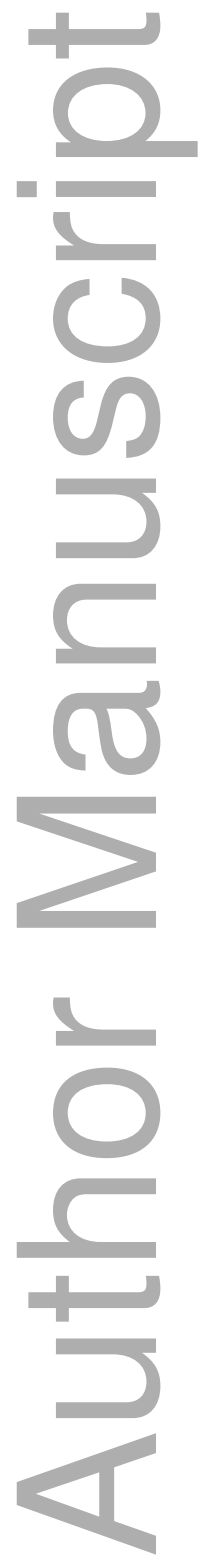




\section{Tables}

\begin{tabular}{lccccccccc}
\hline \hline & \multicolumn{8}{c}{ Coefficient of determination, $\mathrm{r}^{2}$} \\
\cline { 2 - 10 } NUFFT & USF=2 & USF=3 & USF=4 & USF=5 & USF=6 & USF=7 & USF=8 & USF=9 & USF=10 \\
\cline { 2 - 10 } Wavelet & 0.967 & 0.916 & 0.88 & 0.843 & 0.807 & 0.776 & 0.745 & 0.712 & 0.682 \\
DCT & 0.956 & 0.947 & 0.932 & 0.916 & 0.902 & 0.894 & 0.889 & 0.885 & 0.879 \\
Identity & 0.956 & 0.917 & 0.908 & 0.886 & 0.893 & 0.886 & 0.875 & 0.871 \\
\hline \hline
\end{tabular}

TABLE 1 Coefficient of determination, $r^{2}$, of linear fits in Figure 2.

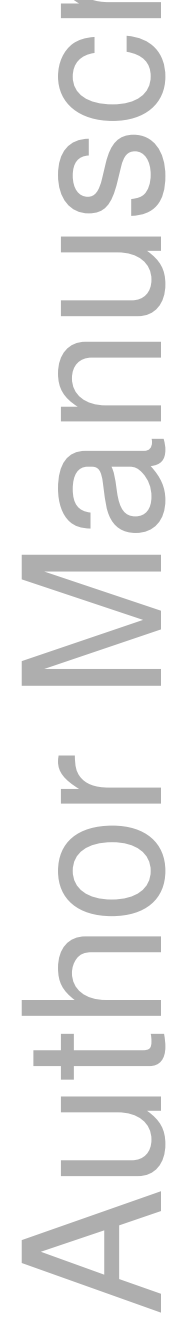

This article is protected by copyright. All rights reserved 


\section{Figures}

Figure 1 CS reconstruction analysis. Structural similarity results of in vivo CS reconstruction across undersampling factors, TV and sparsity weighting in investigated sparsity domains. Red dotted line surrounds area with a higher quality score than NUFFT-based reconstructions at the respective undersampling factor. USF, undersampling factor; DCT, Discrete Cosine Transform; TV, total variation

Figure 2 CS intensity reconstruction performance. Voxelwise comparison between reference image intensity $I_{\text {ref }}$ and undersampled reconstruction intensities $I_{u}$ for all undersampled reconstruction schemes. (a) Point cloud overlay of reconstruction accuracy for all USFs; black dashed line highlights a perfect correlation. (b) Linear least-square fits through point clouds at each USF. (c) Residuals of linear fit results. USF, undersampling factor; $I_{\text {ref }}$, reference image; $I_{u}$, undersampled image; DCT, Discrete Cosine Transform

Figure 3 Investigation of non-linearity in intensity reconstruction. Mean intensity of voxel groups resulting from subdivision of $I_{\text {ref }}$ into 200 equally-spaced intensity groups. For each intensity voxel group, mean intensity values in $\mathrm{I}_{\mathrm{u}}$ over the mean intensity values in $\mathrm{I}_{\mathrm{ref}}$ are plotted across all USFs. Red solid box and blue dashed box show zoomed in view for lower and upper $15 \%$ of intensity groups, respectively. USF, undersampling factor; $I_{\text {ref }}$, reference image; $\mathrm{I}_{\mathrm{u}}$, undersampled image; DCT, Discrete Cosine Transform

Figure 4 TSC estimation results. Top row: Mean TSC estimates in GM (left) and WM (right) for CS and NUFFT-based $I_{u}$ at all USFs. Horizontal black dashed line indicates TSC estimate of $I_{\text {ref. }}$ Bottom row: Relative percentage discrepancy between TSC estimates in $\mathrm{I}_{\mathrm{ref}}$ and $\mathrm{I}_{\mathrm{u}}$. TSC, Tissue sodium concentration; USF, undersampling factor; NUFFT, Non-uniform Fast Fourier Transform; DCT, Discrete Cosine Transform

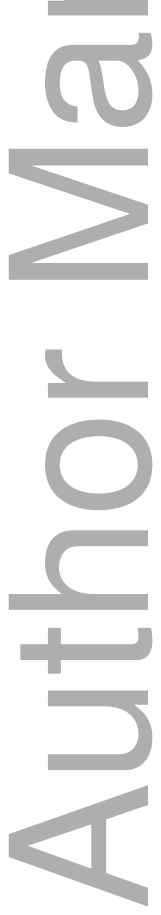




\section{Supporting Material}

Figure S.1 In vivo reconstruction. Axial and sagittal cross section of in vivo reconstructions. Top row: Fully sampled (10,000 projections) reconstruction ( $\left.I_{\text {ref }}\right)$; lower rows: NUFFT and CS reconstructions across different undersampling factors. CS reconstructions with highest sSim score are displayed. USF, undersampling factor; DCT, Discrete Cosine Transform, I $I_{\text {ref }}$, reference image

Figure S.2 Density-colored scatter plots of intensity reconstruction in $I_{\text {ref }}$ and $I_{u}$ in one subject. Matrices display reconstruction correlation across all undersampled reconstruction schemes (rows) and USFs (columns). Black solid line shows identity. Reconstruction performance is illustrated in (a) the extracted brain mask, i.e. structural components, and (b) a background region, i.e. noise/undersampling artifacts. The red dotted line in (a) illustrates a linear leastsquares fit through the point cloud. USF, undersampling factor; $I_{\text {ref }}$, reference image; $I_{u}$, undersampled image; DCT, Discrete Cosine Transform

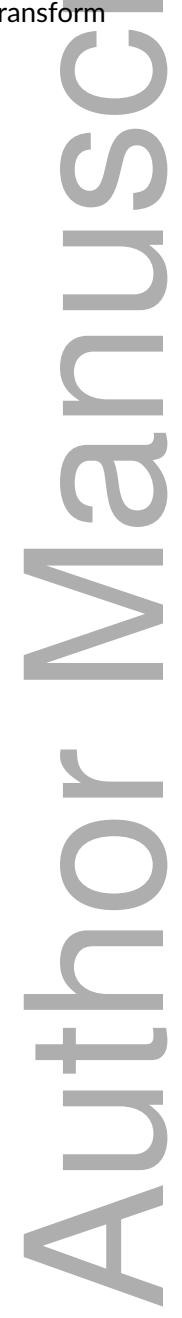




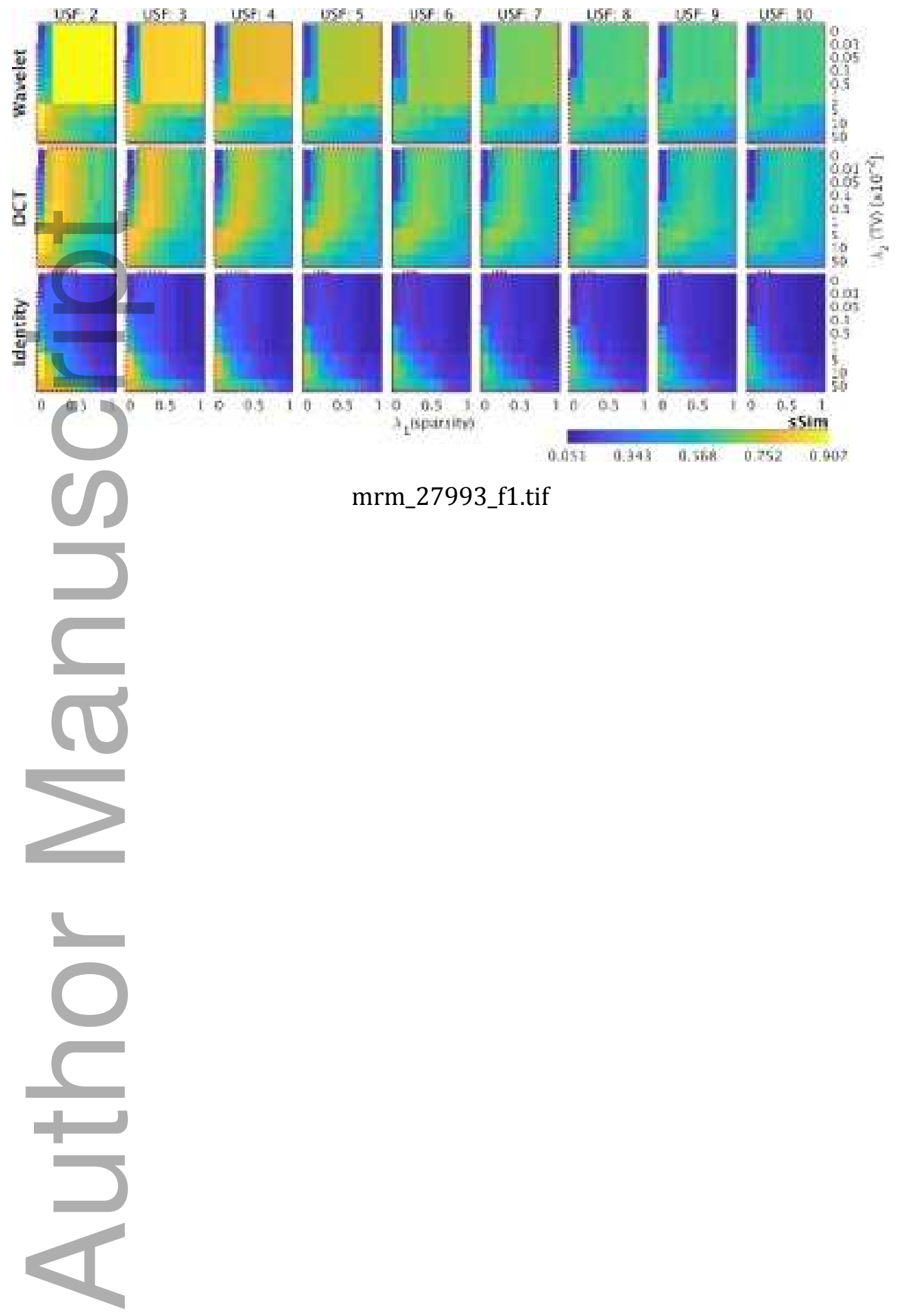



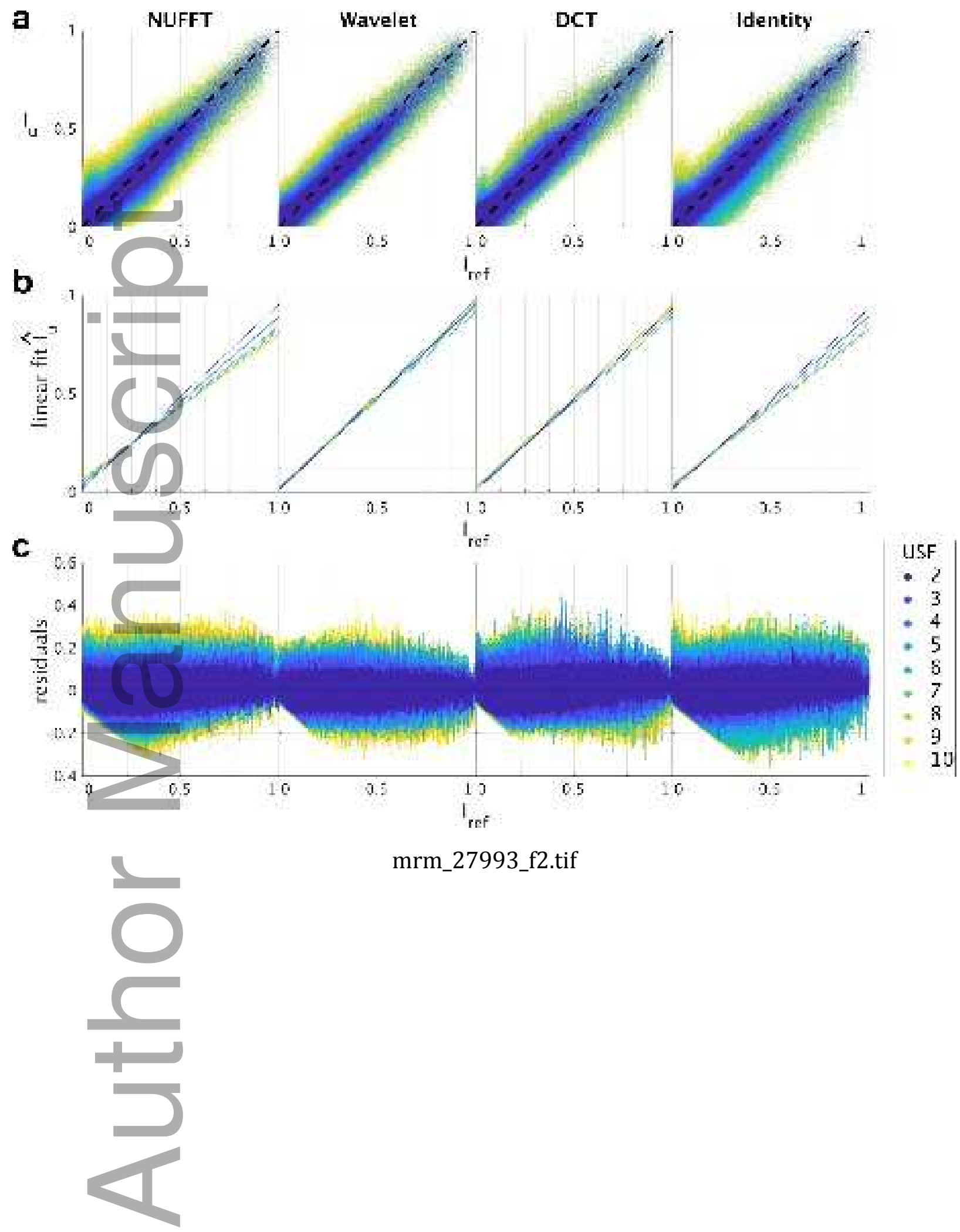

mrm_27993_f2.tif 

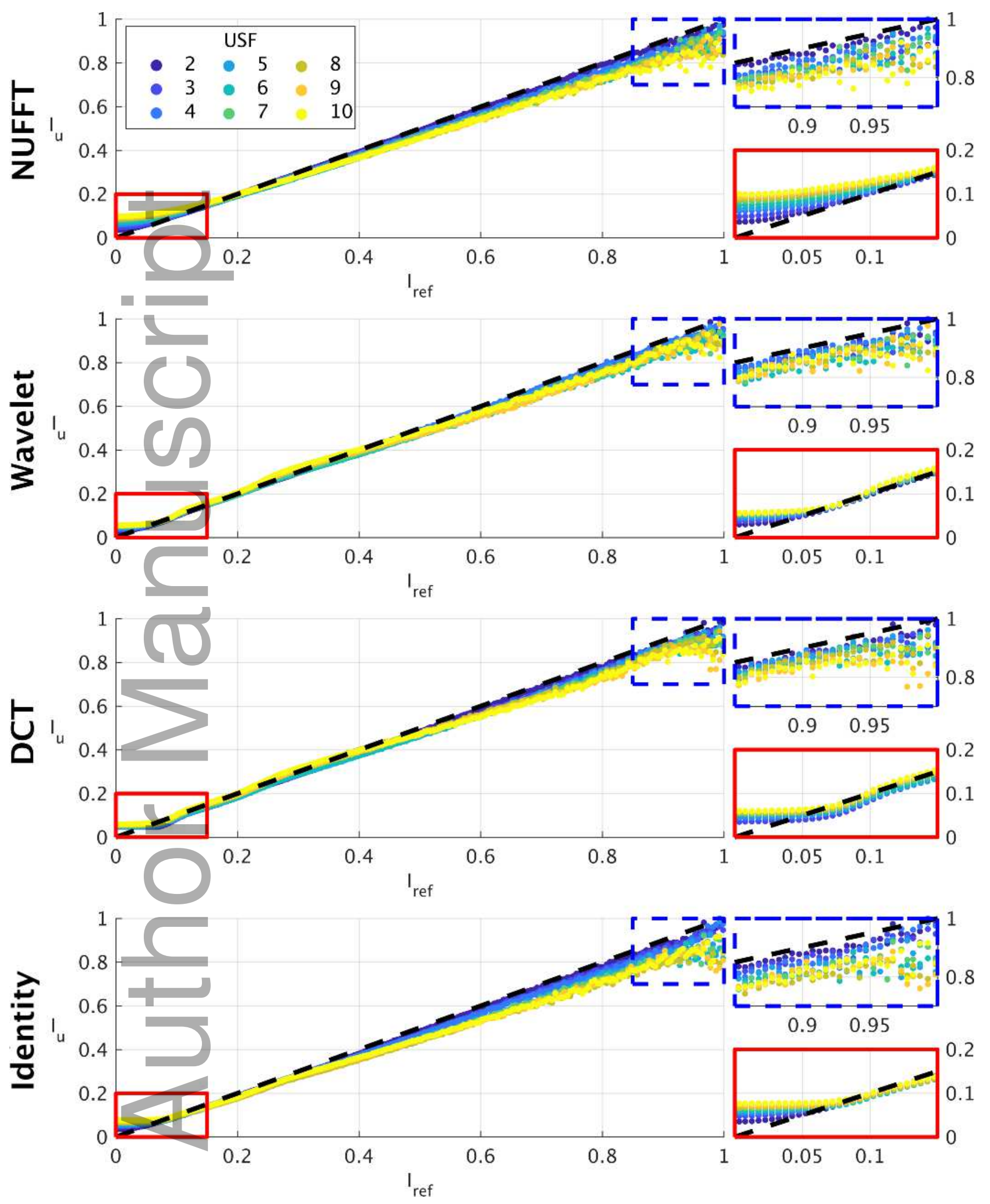

mrm_27993_f3.tif

This article is protected by copyright. All rights reserved 


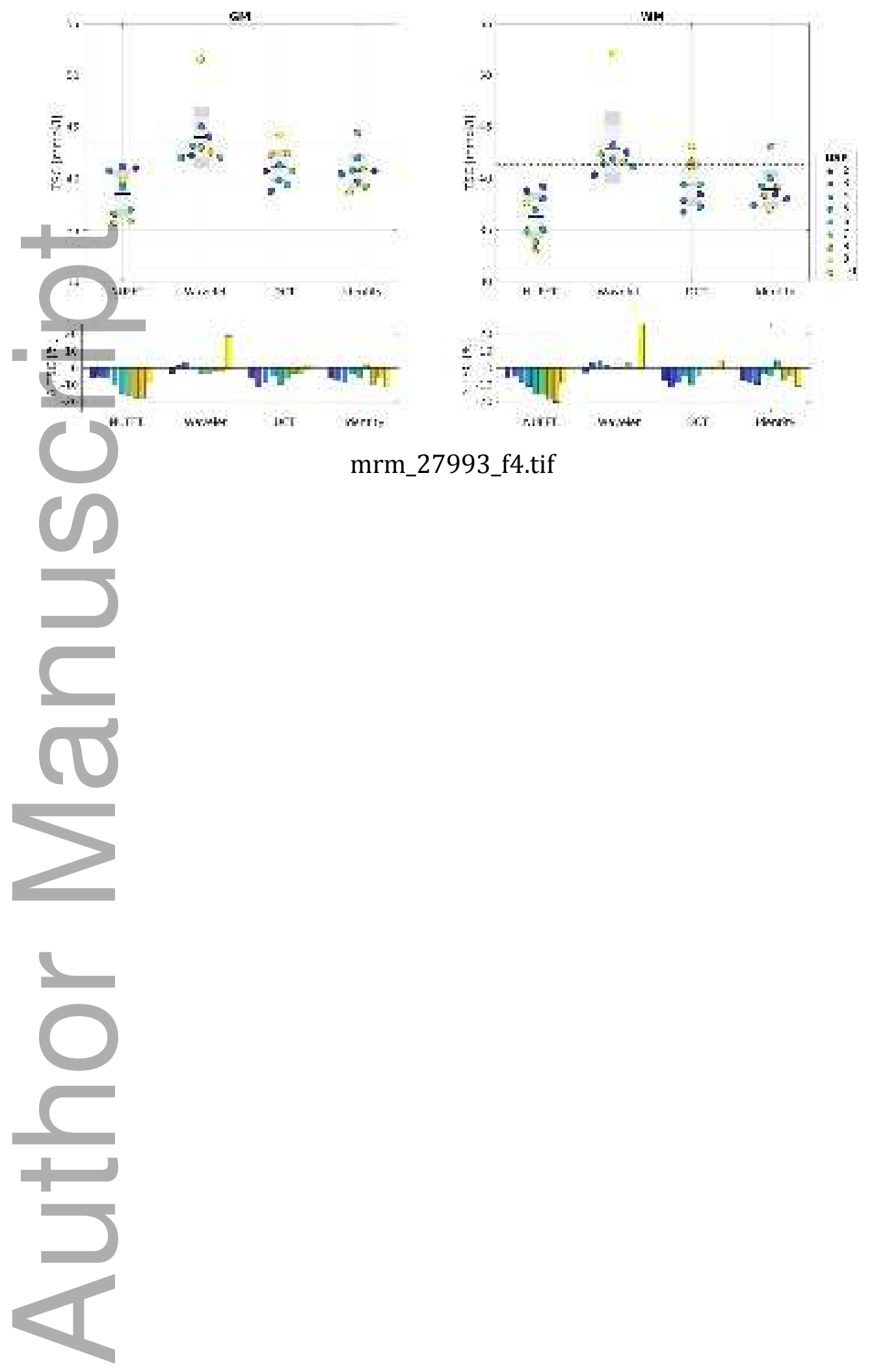

This article is protected by copyright. All rights reserved 


\section{University Library}

\section{- M M I N E R VA A gateway to Melbourne's research publications}

Minerva Access is the Institutional Repository of The University of Melbourne

Author/s:

Blunck, Y;Kolbe, SC;Moffat, BA;Ordidge, RJ;Cleary, JO;Johnston, LA

Title:

Compressed sensing effects on quantitative analysis of undersampled human brain sodium $\mathrm{MRI}$

Date:

2020-03

Citation:

Blunck, Y., Kolbe, S. C., Moffat, B. A., Ordidge, R. J., Cleary, J. O. \& Johnston, L. A. (2020). Compressed sensing effects on quantitative analysis of undersampled human brain sodium MRI. Magnetic Resonance in Medicine, 83 (3), pp.1025-1033. https://doi.org/10.1002/ mrm.27993.

Persistent Link:

http://hdl.handle.net/11343/286401 